\section{Synchrotron Radiation (Production and Properties). By Philip John Duke. Oxford Science Publications, 2000. Pp. 251. Price (hardback) £70.00. ISBN 0198517580.}

Undoubtedly, synchrotron radiation has become a major structural research tool across the world in numerous scientific areas. As a consequence, the number of users of this unique type of radiation has increased considerably, attracting researchers from A(rcheometry) to $\mathrm{Z}$ (oology). Certainly, many of these will not dare to throw light on this black box; however, those who do wonder about the origins of synchrotron radiation will find in P. J. Duke's book a valuable and thorough source of facts describing its physical basis from first principles. From a single free electron to a beam of electrons in a storage ring, from Maxwell's description of electromagnetic radiation to Einstein's special theory of relativity, and from square waves to wiggles, the reader sees the elaborate production and remarkable properties of synchrotron radiation unfolded within merely 251 pages and 15 chapters.

More than 40 years of experience in the field qualify the author to educate an international interdisciplinary community of synchrotron radiation users. While chapters 1-7 deal with essentially the theoretical aspects of radiation emission from accelerated electrons, chapters $8-15$ describe the practical features of electron accelerators. Of course, these are highly relevant to the general properties of the radiation beams which are ultimately produced for research applications, and they are recommended reading, therefore, for those intending to pursue such applications. In addition, these later chapters will provide useful material for those wishing to extend their understanding of modern accelerators for synchrotron radiation.
However, the information that radiates from this book cannot be captured without the appropriate mathematical background and - as one immediately notices - there are plenty of formulae. This may perhaps present an obstacle or even deterrent for the enthusiast with modest mathematical ambitions. A typical synchrotron radiation user without a background in physics is likely to find it hard going. On the other hand, the author attempts to derive most of the equations within the consecutive chapters of the book (i.e. one reason why the first chapter introduces the mathematical picture of electromagnetic waves) and hence the reader is guided without having to resort to much ink and paper or specialized literature. The first four chapters provide a good introduction for physics undergraduates and the rest of the book would be of more interest to graduates aiming to become specialists in synchrotron radiation.

Bearing in mind this specialist viewpoint as well as the price ( $£ 70$ as hardback), it is questionable whether this volume of the series will end up in the top ten list of books for the typical synchrotron radiation user. Certainly, advanced physics students and those involved in beamline design for new synchrotron radiation sources will greatly benefit from the detailed discussions of the concepts and properties of X-ray and electron beams. Without doubt, it ought to be available on bookshelves of science libraries.

\section{J. G. Grossmann \\ R. W. Strange \\ V. P. Suller}

Synchrotron Radiation Department

CLRC Daresbury Laboratory

Warrington WA4 $4 A D$

UK 